\title{
A Numerical and Experimental Study of the Aerodynamics and Stability of a Horizontal Parachute
}

\author{
Mazyar Dawoodian, ${ }^{1}$ Abdolrahman Dadvand, ${ }^{2}$ and Amir Hassanzadeh ${ }^{3}$ \\ ${ }^{1}$ Department of Mechanical Engineering, I. A. University of Takestan, Takestan, Iran \\ ${ }^{2}$ Department of Mechanical Engineering, Urmia University of Technology, Urmia, Iran \\ ${ }^{3}$ Department of Mechanical Engineering, Urmia University, Urmia, Iran
}

Correspondence should be addressed to Mazyar Dawoodian; mazyar_dawoodian@yahoo.com

Received 24 May 2013; Accepted 26 June 2013

Academic Editors: J. López-Puente and R. K. Sharma

Copyright (C) 2013 Mazyar Dawoodian et al. This is an open access article distributed under the Creative Commons Attribution License, which permits unrestricted use, distribution, and reproduction in any medium, provided the original work is properly cited.

\begin{abstract}
The flow past a parachute with and without a vent hole at the top is studied both experimentally and numerically. The effects of Reynolds number and vent ratio on the flow behaviour as well as on the drag coefficient are examined. The experiments were carried out under free-flow conditions. In the numerical simulations, the flow was considered as unsteady and turbulent and was modelled using the standard $k-\varepsilon$ turbulence model. The experimental results reveal good agreement with the numerical ones. In both the experiments and numerical simulations, the Reynolds number was varied from 85539 to 357250 and the vent ratio was increased from zero to $20 \%$. The results show that the drag coefficient decreases by increasing the Reynolds number for all the cases tested. In addition, it was found that at low and high Reynolds numbers, the parachutes, respectively, with $4 \%$ vent ratio and without vent are deemed more efficient. One important result of the present work is related to the effect of vent ratio on the stability of the parachute.
\end{abstract}

\section{Introduction}

The study of bluff bodies involves the consideration of complex aerodynamic phenomena such as semiwake and vortex shedding. The analysis of parachute dynamics is one of the most interesting problems, which would lie within this context. One important feature that may distinguish the computational fluid dynamics (CFD) modelling from the experimental analysis of the parachute behaviour is the geometry flexibility in the latter allowing large variations in the experiments [1]. A parachute is generally equipped with a venthole at the apex to allow some air to flow through the open canopy. The canopy can be represented by a hemispherical shell, which has been shown schematically in Figure 1. In the numerical simulations carried out in the present work, the canopy is considered solid and its direction of motion is from left to right (i.e., the flow direction is from right to left in the positive $x$ direction). The flow structure near the wake of canopy is responsible for the aerodynamic forces and moments it experiences. The relationship between the Reynolds number and flow field structure around spherical bodies in incompressible flow regime has been studied extensively. The numerical study carried out by Natarajan and Acrivos [2] indicated that the wake of a sphere became unstable at a Reynolds number of 105. The experimental results of Sakamoto and Haniu [3] showed that the hairpinshaped vortices begin to be periodically shading when the Reynolds number reaches about 350 . Their research indicated that when the Reynolds number exceeds 6000 , the vortex sheet separating from the surface of the sphere becomes completely turbulent.

According to the experimental work of Bakic and Peric [4] in wind tunnel and water channel, for the Reynolds numbers between 22000 and 50000, the near-wake recirculation region was observed to be large and the wake would perform a progressive wave motion. They also found that as the Reynolds number was increased from 22000 to 350000 , the boundary layer separation point would move from about $82^{\circ}$ to $132^{\circ}$. 


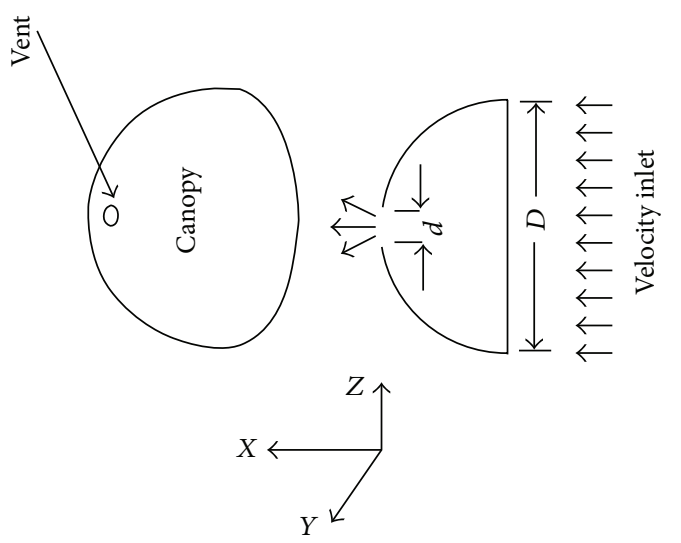

FIGURE 1: Hemispherical shell with a vent in the Cartesian coordinates system.

A circular disk can be regarded as a good representative of round parachutes since the separation line is fixed at the disk edge. Fuchs et al. [5] and Berger et al. [6] have studied the flow structure around the disk and identified three instability modes: an axisymmetric oscillation of the recirculation bubble at a low Strouhal number $(\approx 0.05)$, a low-frequency mode at a Strouhal number of about 0.14 , and a high-frequency mode at a Strouhal number equals to 1.6. The main recirculation bubble extends to about 2.5 diameters behind the disk. Flow visualization of Higuchi [7] confirmed the primary helical mode at a Reynolds number of 104 in the disk wake. However, close-up observations in a water tunnel revealed that the tilting of vortex rings results in asymmetric structures after the first diameter in the disk wake. Peterson et al. [8] have studied the characteristic features of parachute canopies during various stages from breathing to final developing.

Experimental investigation of the drag forces on flexible rectangular canopies was conducted by Filippone [9]. He showed that the drag coefficient decreased weakly by increasing the Reynolds number to 2000000 and decreased intensively by increasing the ratio of perforated tape area to plan form area. Izadi and Mohammadizadeh [10] and Izadi and Dawoodian [11] have numerically studied the Relationship between the drag coefficient and the vent diameter. Takizawa et al. [12] performed a comprehensive numerical calculation on the fluid-structure interaction (FSI) modelling of clusters of ringsail parachutes. They also presented a brief stability and accuracy analysis for the deformingspatial-domain/stabilized space-time (DSD/SST) formulation, which is the core numerical technology of the SSTFSI technique. Cao and Jiang [13] calculated the drag coefficient for a parachute using a finite volume method and SpalartAllmaras turbulence model. The separation flow, which truthfully reflects the characteristics during the terminal descent, accords with the fluid dynamics rules in their study. Cao and $\mathrm{Xu}$ [14] analyzed canopy shape and the parameters of parachute inflation by means of establishing parachute's flying physical model through four methods (i.e., tiny segment analysis method, inflating distance method, moment method and simulating canopy shape method). They concluded that moment method, and simulating canopy shape method are only fitted to the main phase of inflating in theory and inflating distance method can be applied to evaluate the dynamic load of parachute inflation.

A complete understanding of the flow physics around a parachute is still one of the most scientific challenging issues from both the theoretical and experimental viewpoints. Recent efforts that have successfully benefited from using CFD simulations could explain the basic principles behind this phenomenon. Thus far, a comprehensive research has not been performed to study the variations of drag coefficient of the flexible canopies with the Reynolds number and vent ratios. In this paper, the experimental tests and numerical simulations are conducted to obtain the drag coefficient on a hemispherical shell for different Reynolds numbers and vent ratios. The venthole is located at the shell apex. The main purpose is to find the effects of the Reynolds number and vent diameter on the drag coefficient as well as on the flow field. These findings may be useful in parachute designing and its applications.

\section{Experimental Setup}

The experiments were conducted under the free-flow conditions (i.e., at the atmospheric pressure and temperature). The test rig was mounted on a vehicle, which moved with the speed of 8.3 to $33.3 \mathrm{~m} / \mathrm{s}$. The inflated canopy setup mounted on the vehicle along with the supporting hardware is shown in Figure 2 schematically. The parachute assembly was positioned in a horizontal orientation. The canopy was attached to a stationary support by six threads. The forebody had a diameter of $1.4 \mathrm{~cm}$ and a length of $2 \mathrm{~m}$. The crosssectional area of the support was less than $2 \%$ of the projected area of the canopy.

By streamlining the support and reducing its diameter, the wakes around the support were kept to a minimum. Flow visualization confirmed that the wake of support rods had a negligible effect on the dynamics of the parachute canopy. The vent ratio of the parachute is varied from $0 \%$ to $20 \%$ (i.e., $d / D=0-0.2$ ). The Reynolds number for a parachute canopy is commonly based on the diameter $D$. In this research, the Reynolds number ranges from 85539 to 357250. A dynamometer was used to record the force at every stage of the experiment. Figure 3 displays two typical parachutes with a venthole used in the experiments.

\section{Numerical Simulation}

3.1. Governing Equations. For the external flows around spherical obstacles, the critical Reynolds number is about 800 , beyond which the wake flow becomes turbulent [15]. Since all the Reynolds numbers considered in the present work are much greater than this critical Reynolds, the flow around the parachute is assumed to be turbulent. Therefore, the three-dimensional unsteady Reynolds-averaged NavierStokes (RANS) equations combined with the standard $k-\varepsilon$ turbulence model are employed to simulate the flow around 


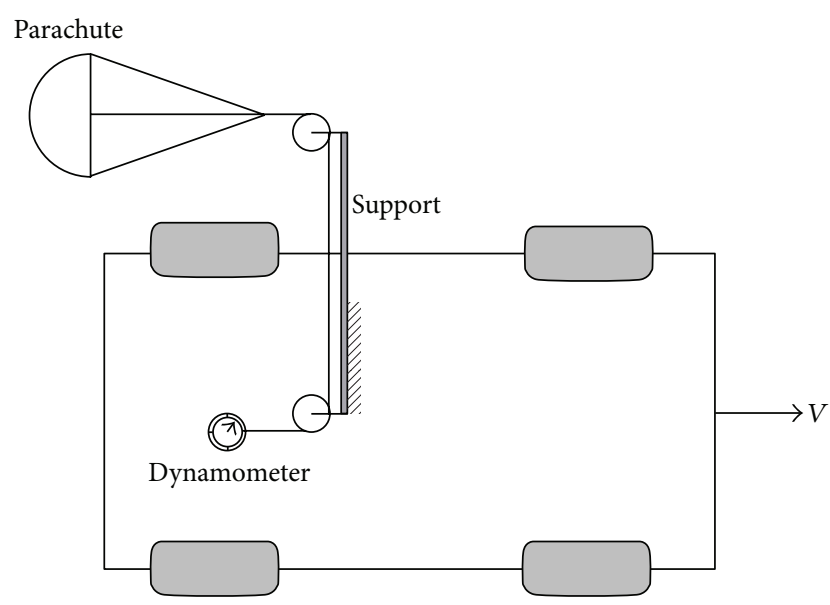

FIgURE 2: Top view of the experimental setup (not to scale).

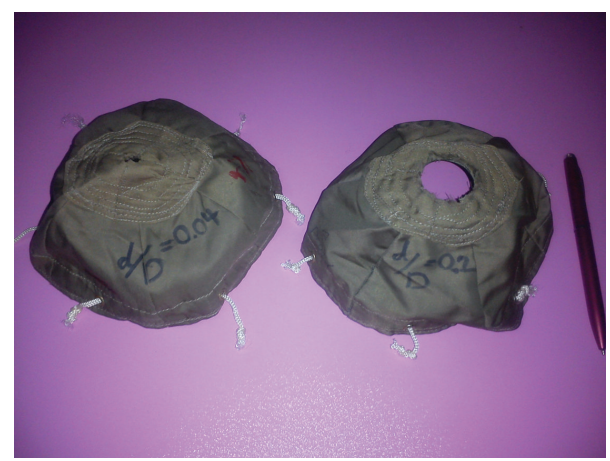

Figure 3: Two typical parachutes utilized in the present experiments.

the parachute. It may be noted that the RNG $k$ - $\varepsilon$ turbulence model and more advanced turbulence models such as the Reynolds stress equations were also investigated, but these models could not give good numerical results. Since the experiments were performed under the free-flow conditions, the parachute would not have a significant effect on the temperature of air; so, in the simulations the density of air is assumed constant. In addition, since the minimum and maximum Mach numbers are equal, respectively, to 0.033 and 0.137 , the flow was considered incompressible. The governing RANS equations (i.e., the continuity and momentum equations) are given as

$$
\begin{gathered}
\frac{\partial \bar{u}_{i}}{\partial x_{i}}=0 \\
\rho\left(\frac{\partial \bar{u}_{i}}{\partial t}+\bar{u}_{j} \frac{\partial \bar{u}_{i}}{\partial x_{j}}\right)=-\frac{\partial \bar{p}}{\partial x_{i}}+\bar{B}_{i}+\frac{\partial}{\partial x_{j}}\left(\mu \frac{\partial \bar{u}_{i}}{\partial x_{j}}-\rho \bar{u}_{i}^{\prime} \bar{u}_{j}^{\prime}\right) .
\end{gathered}
$$

$\bar{B}_{i}$ is the body force(s) in the $i$ th direction. The normal velocity on all surfaces of the body is considered zero.
The turbulence kinetic energy $k$ and its rate of dissipation $\varepsilon$ are obtained from the following transport equations:

$$
\begin{aligned}
\rho \frac{\partial k}{\partial t}+\rho\left(u_{j} \frac{\partial k}{\partial x_{j}}\right)= & \frac{\partial}{\partial x_{j}}\left[\left(\mu+\frac{\mu_{t}}{\sigma_{k}}\right) \frac{\partial k}{\partial x_{j}}\right]+G_{k} \\
& +G_{b}-\rho \varepsilon-Y_{M}, \\
\rho \frac{\partial \varepsilon}{\partial t}+\rho\left(u_{j} \frac{\partial \varepsilon}{\partial x_{j}}\right)= & \frac{\partial}{\partial x_{j}}\left[\left(\mu+\frac{\mu_{t}}{\sigma_{\varepsilon}}\right) \frac{\partial \varepsilon}{\partial x_{j}}\right] \\
& +C_{1 \varepsilon} \frac{\varepsilon}{k}\left(G_{k}+C_{3 \varepsilon} G_{b}\right)-C_{2 \varepsilon} \rho \frac{\varepsilon^{2}}{k} .
\end{aligned}
$$

In these equations, $G_{k}, G_{b}$, and $Y_{M}$ represent the generation of turbulence kinetic energy due to the mean velocity gradients, the generation of turbulence kinetic energy due to buoyancy, and the contribution of the fluctuating dilatation in compressible turbulence to the overall dissipation rate, respectively. $C_{1 \varepsilon}$ and $C_{2 \varepsilon}$ are constants. $\sigma_{k}$ and $\sigma_{\varepsilon}$ are the turbulent Prandtl numbers for $k$ and $\varepsilon$, respectively. The turbulent (or eddy) viscosity $\mu_{t}$ is computed as follows:

$$
\mu_{t}=\rho C_{\mu} \frac{k^{2}}{\varepsilon}
$$

where $C_{\mu}$ is a constant. The constants $C_{1 \varepsilon}, C_{2 \varepsilon}, C_{\mu}, \sigma_{k}$, and $\sigma_{\varepsilon}$ take the following values: $C_{1 \varepsilon}=1.44, C_{2 \varepsilon}=1.92, C_{\mu}=0.09$, $\sigma_{k}=1.0$, and $\sigma_{\varepsilon}=1.3$.

The flow equations, described above, are solved by FLUENT's pressure-based segregated solver. It is worth mentioning that when $\mathrm{Re}>10^{3}$, the inertial forces dominate and the drag force is approximately proportional to the square of the velocity; that is, $F_{d}=0.5 \rho C_{d} A U_{\infty}^{2}$.

Here $C_{d}$ is the drag coefficient defined as

$$
C_{d}=\frac{F_{d}}{0.5 \rho A U_{\infty}^{2}},
$$

where $A=\left(\pi D^{2} / 4\right)$ is the projected area of the hemispherical cup and $U_{\infty}$ is the free stream velocity. The fluid properties are considered at standard condition. Therefore the air density $\rho$ and viscosity $\mu$ are taken as $1.25 \mathrm{~kg} \cdot \mathrm{m}^{-3}$ and $1.78 \times 10^{-5} \mathrm{~kg} \cdot \mathrm{m}^{-1} \cdot \mathrm{s}^{-1}$, respectively. These values are kept constant during the simulation process.

3.2. Numerical Model Description and Boundary Conditions. Figure 4 depicts the 3D-CFD model of the parachute along with the boundary conditions. Note that, in all of the simulations carried out in the present work, the geometrical dimensions of the parachute are kept constant. However, the vent diameter and the Reynolds number are varied in order to study their effect on the drag coefficient as well as on the flow structure. In addition, to link/match similar conditions between the simulations and experiments, the numerical Reynolds numbers are chosen the same as those of the experiments. An unstructured (or irregular) T-Grid mesh with finer mesh points near the parachute body is used. As shown in Figure 5, the computational domain assumes 


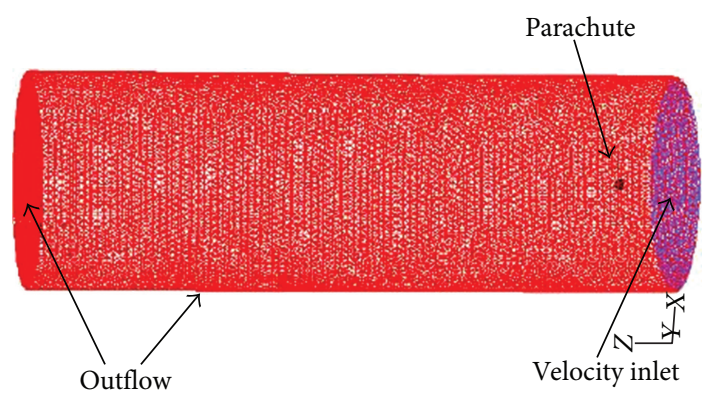

FIGURE 4: CFD mesh points and boundary conditions.

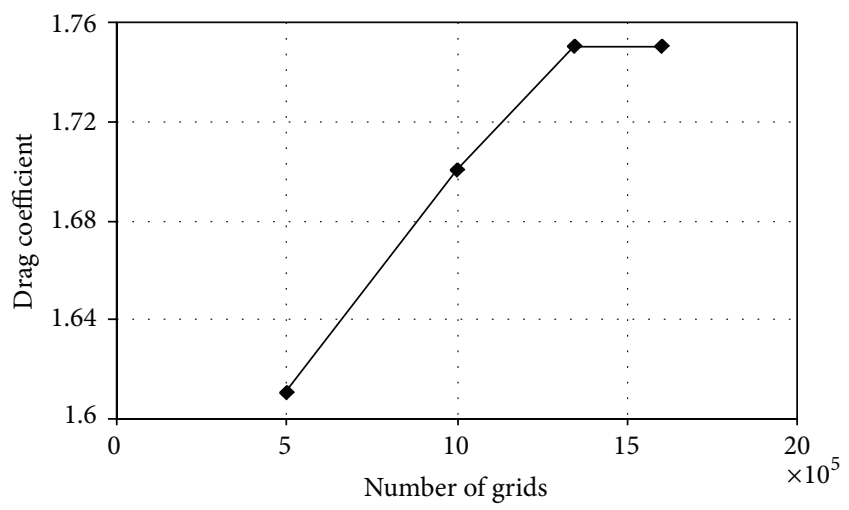

FIGURE 5: Grid size-dependence study of drag coefficient at different mesh densities.

a cylindrical shape, where $\mathrm{A}, \mathrm{B}$, and $\mathrm{C}$ distances are taken as 46,10 , and 10 times the parachute diameter $D$, respectively.

The first order upwind scheme is utilized to discretize the convective terms, and the SIMPLE algorithm is used for solving the governing equations. It must be noted that the calculations are allowed to continue for 8 seconds until the parameters like $C_{d}$ remain constant; that is, the results are associated with the steady state condition. The outflow boundary condition has been applied at both the lateral and outlet boundaries, and the velocity inlet has been considered as the inlet boundary condition. The no-slip boundary condition is applied on the body of the hemispherical cup. The main boundaries are shown in Figure 4.

3.3. Grid Dependence Study. The numerical simulations have been carried out for different grid densities in order to find the grid independent solutions. For this purpose, first the grid independence study has been accomplished for $\mathrm{Re}=85539$ and $d / D=0$. The variations of the drag coefficient as the key parameter against the number of grid cells have been depicted in Figure 5. According to this figure, a grid system of 1344000 tetrahedral cells was found to be sufficient to resolve the details of the flow field. Therefore, nominally a grid system of 1344000 tetrahedral cells was used in all the simulations carried out in the present work.

\section{Results and Discussions}

4.1. Drag Coefficient. In order to investigate the parachute performance under different flow conditions, six parachutes with various vent diameter ratios $d / D$ of $0,0.04,0.08$, $0.12,0.16$, and 0.20 each at different Reynolds numbers of $85539,114000,146330,171100,228070,292660$, and 357250 are studied in both the experimental and numerical cases. Figure 6 shows the numerical and experimental drag coefficient $C_{d}$ variations as a function of the Reynolds number for different vent ratios of $0-20 \%$.

It is clear that for all the vent ratios tested, $C_{d}$ decreases by increasing the Reynolds number. In addition, the drag coefficient seems to experience a (relatively) sharp decrease at lower Reynolds numbers, but it takes a smooth change as the Reynolds number increases. One can also observe that the value of $C_{d}$ reaches a constant value and the diagram finds an asymptote. Although the trend of all the diagrams in Figure 6 is roughly the same, this tendency is more evident for $d / D$ $=0,0.12$, and 0.2. There is a good agreement between the experimental and numerical results for all the cases studied. The difference between the maximum and minimum values of $C_{d}$ is about 0.2 , which corresponds to the case with $d / D=$ 0.20 .

Figure 7 illustrates the experimental $C_{d}$ changes versus vent ratio $d / D$ for different Reynolds numbers. The results show that $C_{d}$ decreases by increasing the Reynolds number. This may be attributed to the fact that the formation of the wakes behind the parachute causes the drag force to decrease. In all the Reynolds numbers tested except in the last two ones (i.e., $\mathrm{Re}=292660$ and 357250 ), the parachute with $d / D$ ratio of 0.04 possesses the highest value of the drag coefficient as compared to the other vent ratios. According to this figure, the maximum amount of $C_{d}$ achieved during the experiments is 1.77 , which pertains to $\operatorname{Re}=85539$ and $d / D=0.04$. For the $d / D$ values greater than $0.04, C_{d}$ will be below this value for all the Reynolds numbers tested. Therefore, at high Reynolds numbers it is suggested to use parachutes without vent since such parachutes experience higher $C_{d}$ in comparison with the other cases.

4.2. The Flow Field around the Parachute. Figure 8 shows the velocity vectors related to the unsteady turbulent flow around the parachute obtained from the numerical simulations.

According to this figure, it is evident that for all the vent ratios and the Reynolds numbers tested, two wakes both inside and outside the parachute are observed. In addition, the outer wakes are responsible for increasing the drag force.

The size of these wakes seems to increase moderately as the Reynolds number is increased. In general, the vent ratio is the main cause of the pressure difference decrease across the wall of the parachutes (i.e., between the outer and inner regions of it). In fact, by increasing the vent diameter the outflow flux increases, hence, decreasing the pressure difference between the inner and outer regions of the parachute. 


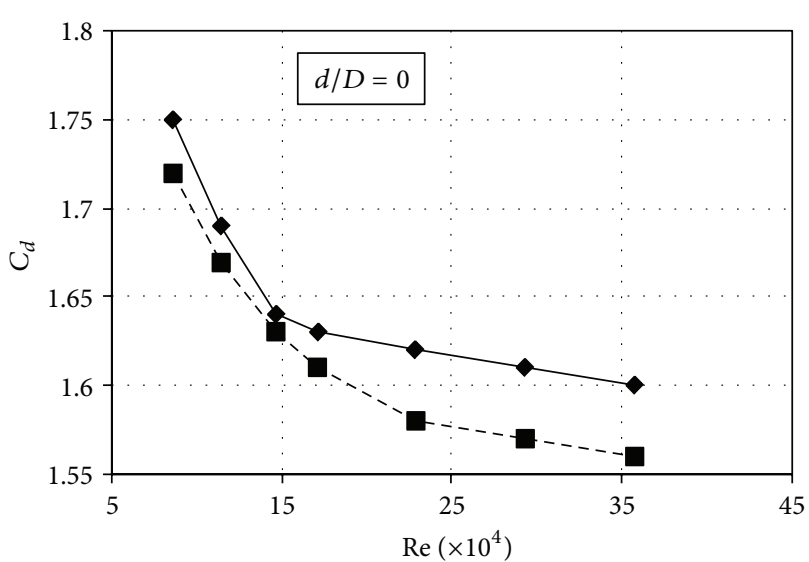

(a)

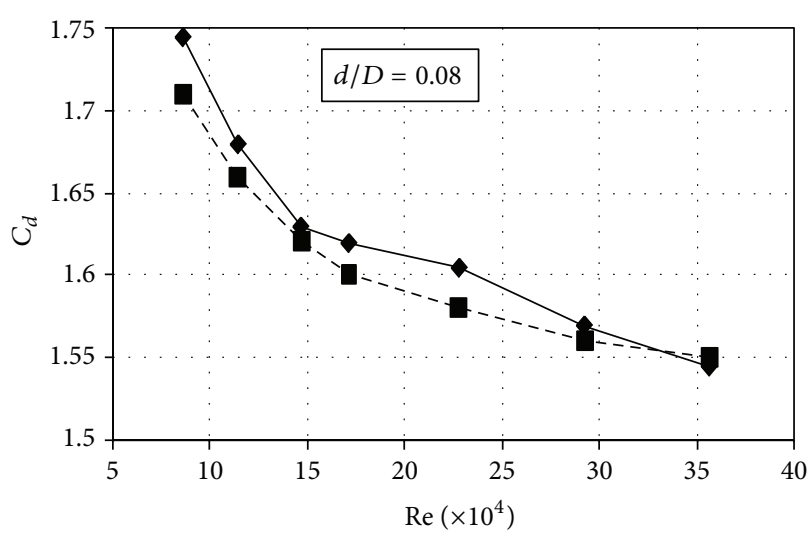

(c)

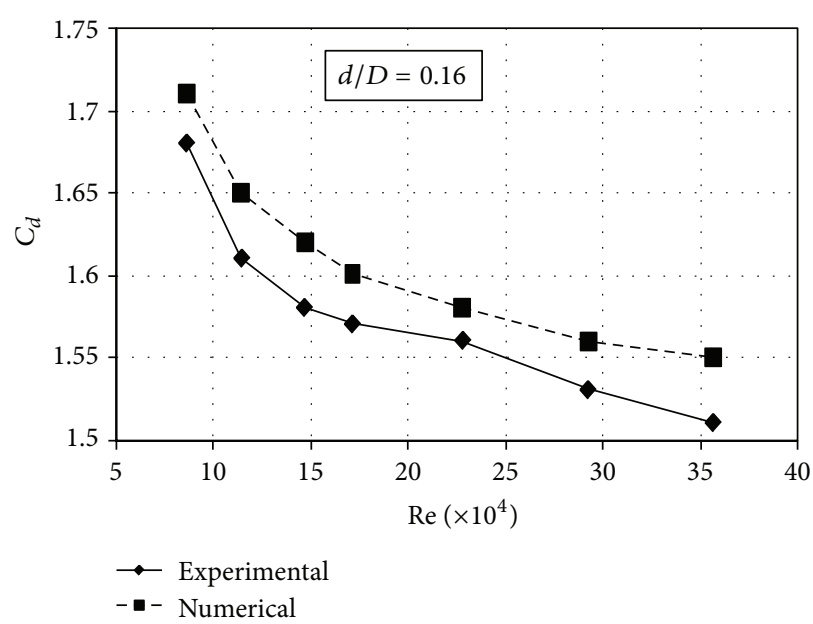

(e)

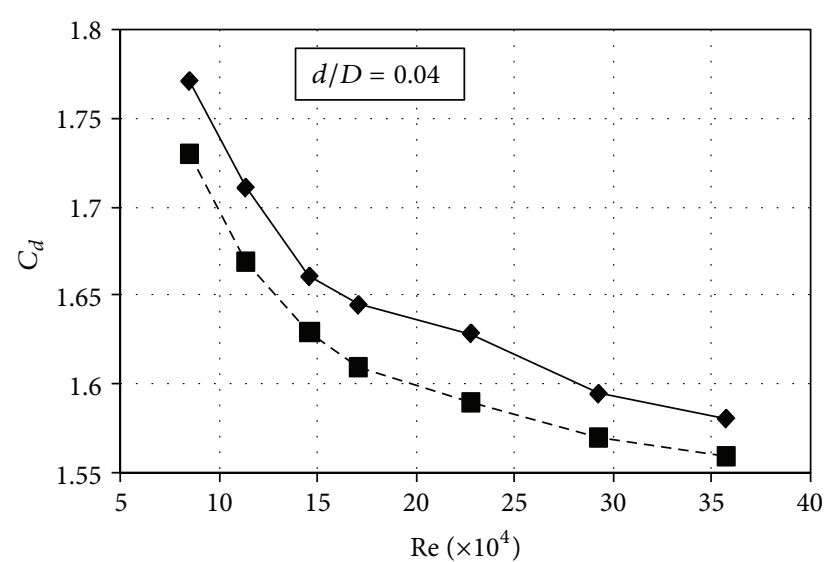

(b)

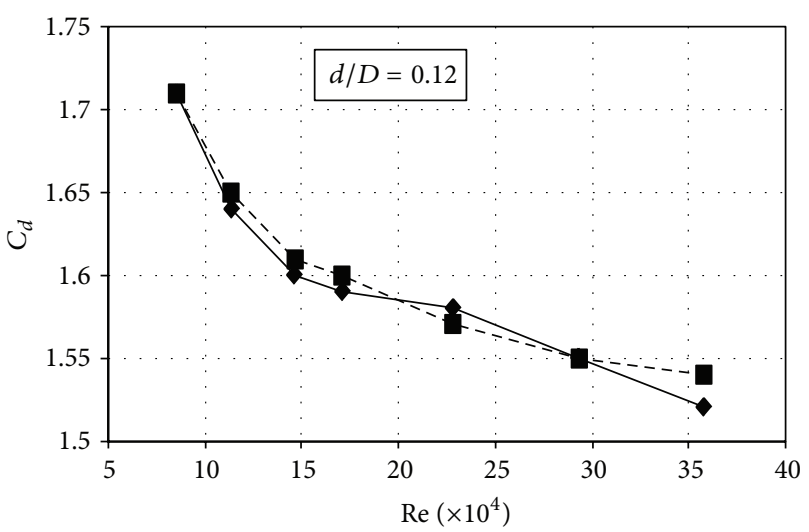

(d)

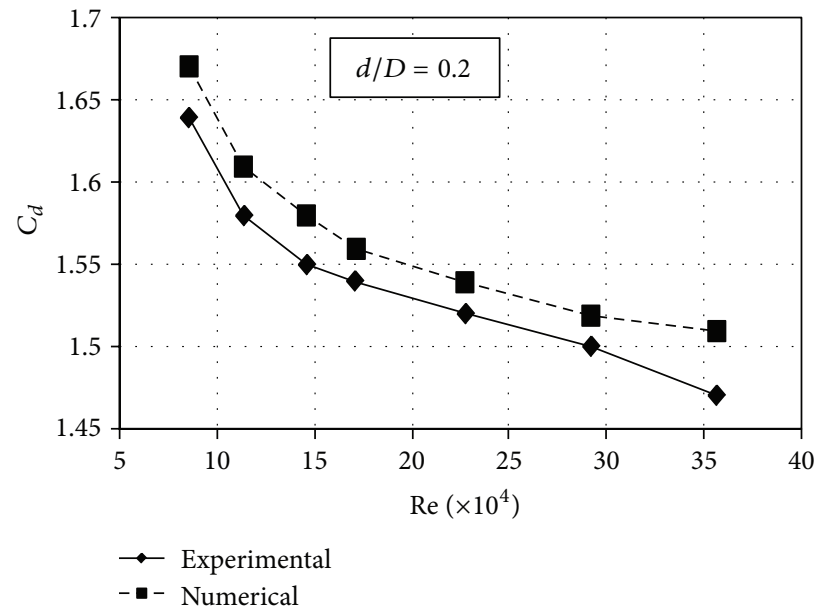

(f)

FIgURE 6: Drag coefficient variations as a function of the Reynolds number for different vent ratios.

4.3. Stability Analysis. During the experimental tests, the parachute was observed to be oscillating when the Reynolds number exceeded some certain values.

At low Reynolds numbers, the parachute was found to be stable without any oscillations. However, as the Reynolds number was increased beyond a certain value, the parachute became unstable. So transitions from stable to unstable condition were observed for all vent ratios. Figure 9 shows the stable and unstable regions observed in the experiments.

It is clear that as the vent diameter is increased, the transition from stable to unstable conditions is delayed. For the parachute without venthole, at $\mathrm{Re}=85539$ no oscillation 


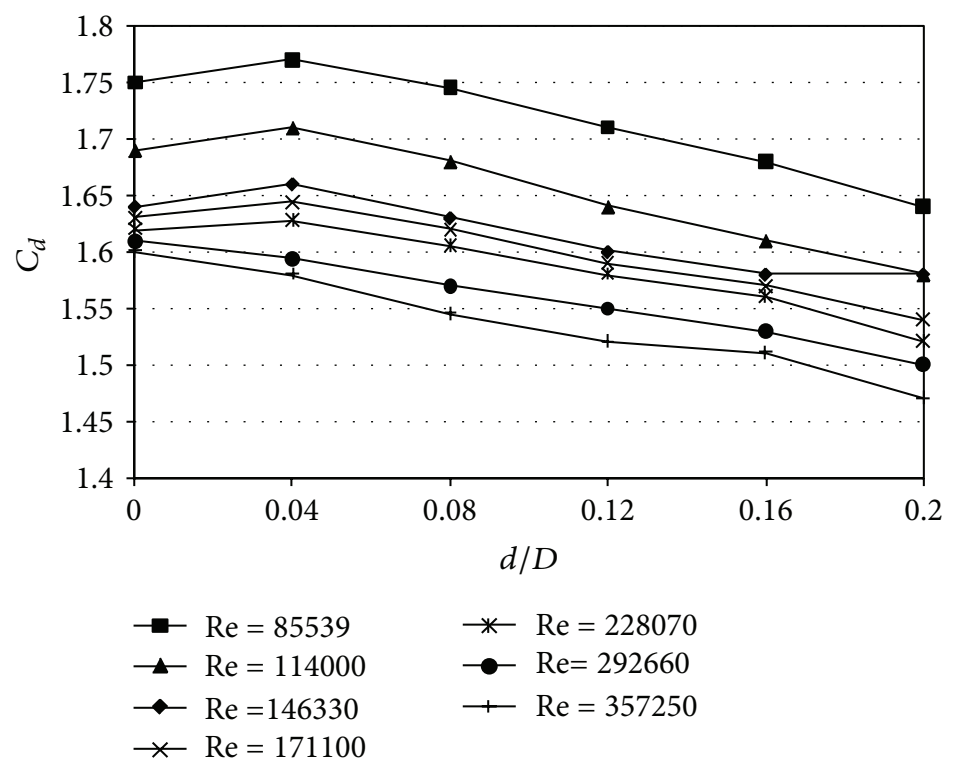

FIGURE 7: Experimental drag coefficient variations against vent ratios for different Reynolds numbers.

is observed. However, for $\mathrm{Re}=114000$ we observed very intensive oscillations. For the parachute with vent of $4 \%$, the oscillations were commenced at $\mathrm{Re}=114000$.

It may be noted that by increasing the vent ratio, the critical Reynolds number at which the parachute started to oscillate is increased. For parachutes with vent ratios of $8 \%$ and $12 \%$, these critical Reynolds numbers are 171100 and 292600 , respectively.

\section{Conclusion}

In this paper, the dynamic behaviour of a parachute is studied both experimentally and numerically. Different cases with various vent ratios are tested at different Reynolds numbers. In the numerical simulations, the flow is considered turbulent and the standard $k-\varepsilon$ turbulence model is used to model the flow field. In addition, an unsteady state condition is considered for the numerical models, but the calculations are continued until the results remain constant. The CFD simulations have been done by using FLUENT software. The effects of the vent ratio and the Reynolds number on the drag coefficient are examined. The results show that for all cases as the Reynolds number is increased the drag coefficient is decreased. In addition, in all the simulations carried out in the present work, two wakes are observed behind the parachute. The maximum value of the drag coefficient measured during the experiments is about 1.77, which is associated with $\mathrm{Re}=$ 85539 and $d / D=0.04$. In addition, at high Reynolds numbers the parachute without vent experiences a higher drag force as compared with the other cases. Comparison between the simulations and experiments evidences good agreements. Finally, the experiments show that the parachute begins oscillating when the Reynolds numbers exceed a certain critical value, which is different for different vent ratios. This would imply that every case has a stable and unstable region. As the vent ratio increases, the oscillations would occur at higher Reynolds numbers.

\section{Abbreviations}

Nomenclature

A: $\quad$ Area $\left(\mathrm{m}^{2}\right)$

$C_{d}$ : Drag coefficient

$d: \quad$ Vent diameter $(\mathrm{m})$

D: Canopy diameter $(\mathrm{m})$

$F_{d}:$ Drag force $(\mathrm{N})$

$k$ : Turbulence kinetic energy $\left(\mathrm{m}^{2} \cdot \mathrm{s}^{-2}\right)$

$M$ : Mach number

p: $\quad$ Pressure $\left(\mathrm{N} \cdot \mathrm{m}^{-2}\right)$

Re: Reynolds number

$U_{\infty}$ : Free stream velocity $\left(\mathrm{m} \cdot \mathrm{s}^{-1}\right)$.

\section{Greek Symbols}

$\varepsilon:$ Turbulence dissipation rate $\left(\mathrm{m}^{2} \mathrm{~s}^{-3}\right)$

$\rho:$ Density $\left(\mathrm{kg} \mathrm{m}^{-3}\right)$

$\mu$ : Dynamic viscosity $\left(\mathrm{kg} \mathrm{m}^{-1} \mathrm{~s}^{-1}\right)$

$\mu_{t}$ : Turbulent viscosity $\left(\mathrm{kg} \mathrm{m}^{-1} \mathrm{~s}^{-1}\right)$.

\section{Subscripts}

$i$ : $\quad$ Direction

max: Maximum

min: Minimum. 


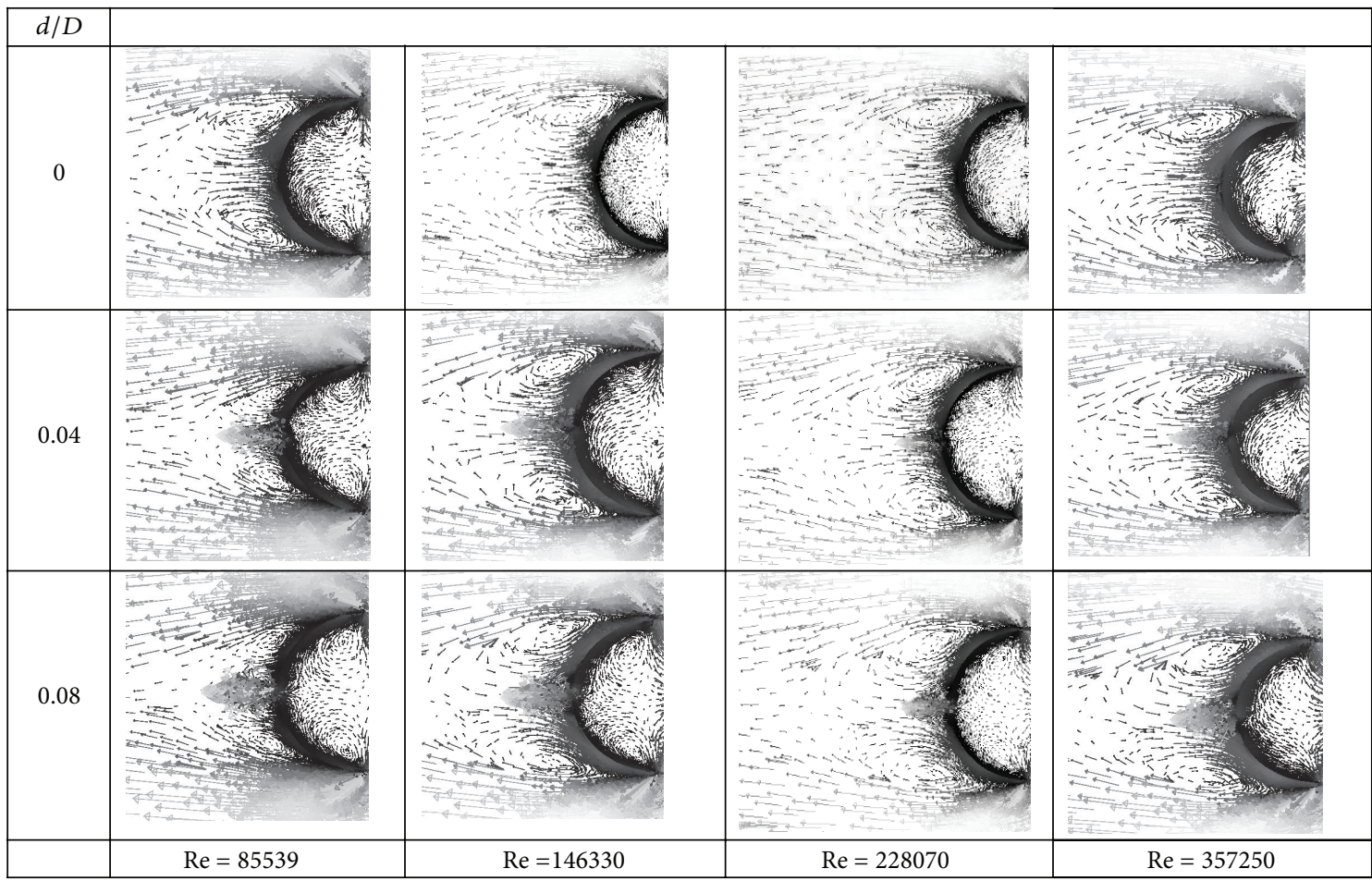

(a)

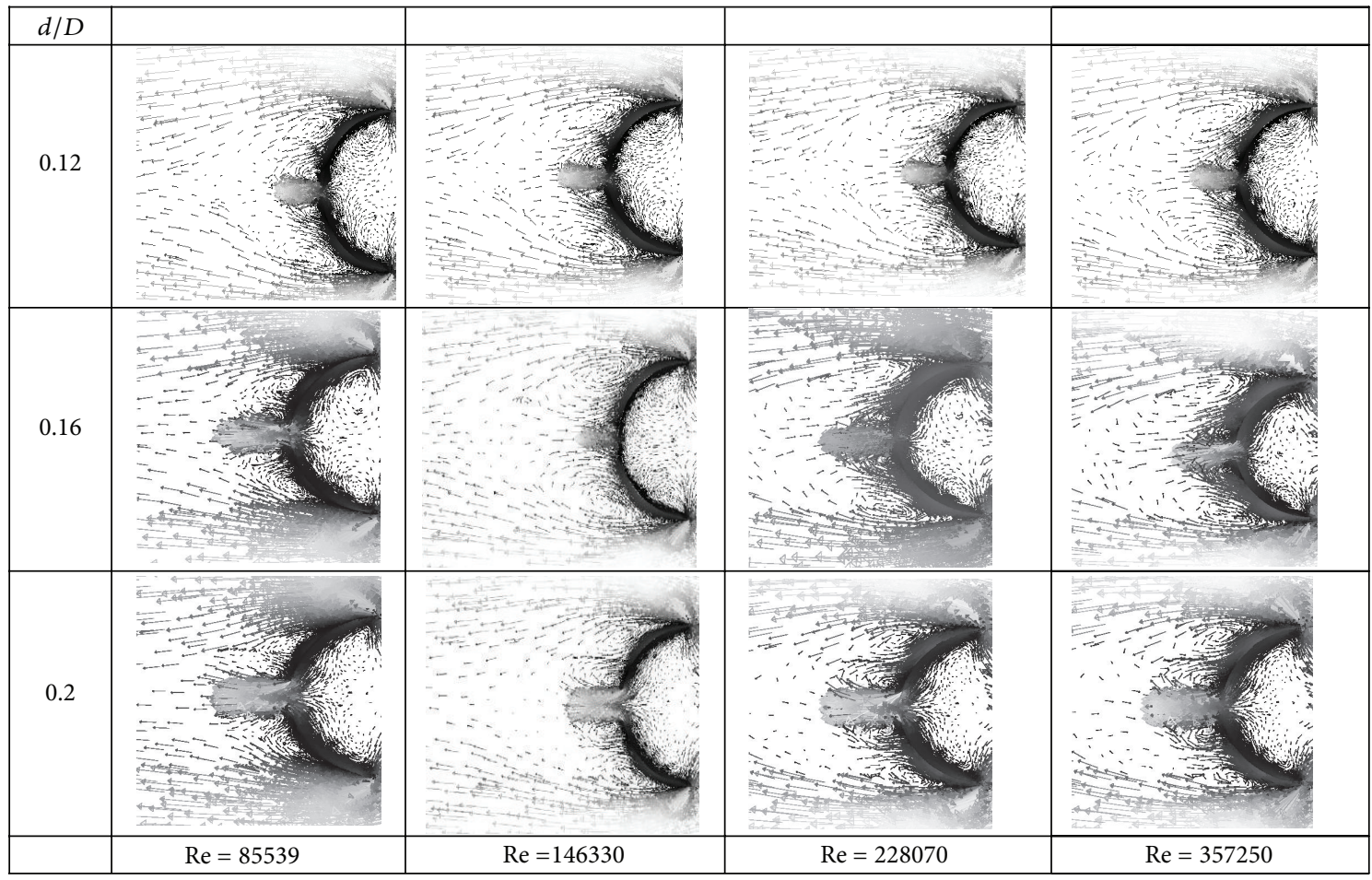

(b)

FIGURE 8: Velocity vectors around a hemispherical shell parachute under different conditions. 


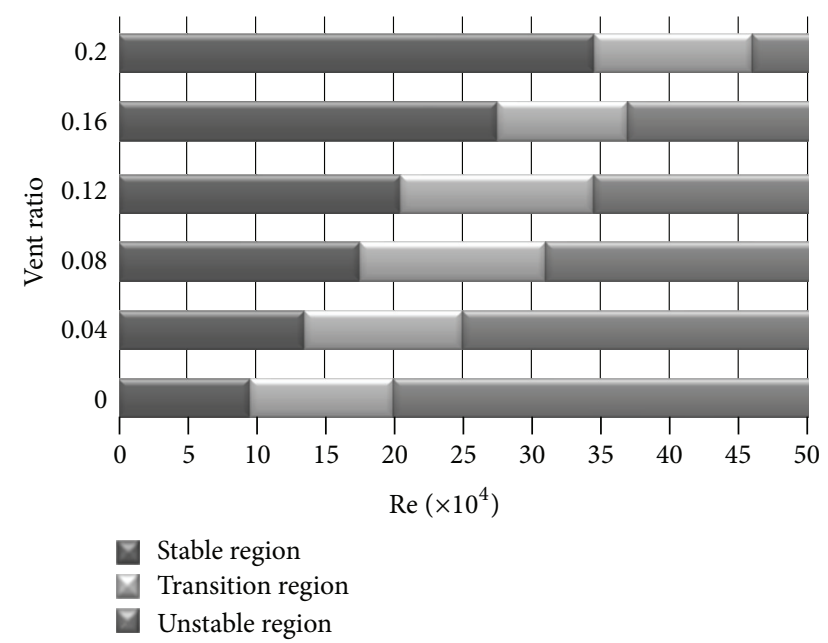

Figure 9: Stable and unstable zones obtained from the experimental analysis.

\section{References}

[1] A. Al Musleh and A. Frendi, "On the effects of a flexible structure on boundary layer stability and transition," Journal of Fluids Engineering, vol. 133, no. 7, Article ID 071103, 6 pages, 2011.

[2] R. Natarajan and A. Acrivos, "The instability of the steady flow past spheres and disks," Journal of Fluid Mechanics, vol. 254, pp. 323-344, 1993.

[3] H. Sakamoto and H. Haniu, "A study on vortex shedding from spheres in a uniform flow," Journal of Fluids Engineering, vol. 112, no. 4, pp. 386-392, 1990.

[4] V. Bakic and M. Peric, "Visualization of flow around sphere for Reynolds numbers between 22,000 and 400,000," Thermophysics and Aeromechanics, vol. 12, no. 3, pp. 307-315, 2005.

[5] H. V. Fuchs, E. Mercker, and U. Michel, "Large-scale coherent structures in the wake of axisymmetric bodies," Journal of Fluid Mechanics, vol. 93, no. 1, pp. 185-207, 1979.

[6] E. Berger, D. Scholz, and M. Schumm, "Coherent vortex structures in thewake of a sphere and a circular disk at rest and under forced vibrations," Journal of Fluids and Structures, vol. 4, no. 3, pp. 231-257, 1990.

[7] H. Higuchi, "Visual study on wakes behind solid and slotted axisymmetric bluff bodies," Journal of Aircraft, vol. 28, no. 7, pp. 427-430, 1991.

[8] C. W. Peterson, J. H. Strickland, and H. Higuchi, "The fluid dynamics of parachute inflation," Annual Review of Fluid Mechanics, vol. 28, pp. 361-387, 1996.

[9] A. Filippone, "On the flutter and drag forces on flexible rectangular canopies in normal flow," Journal of Fluids Engineering, vol. 130, no. 6, Article ID 061203, 8 pages, 2008.

[10] M. J. Izadi and M. Mohammadizadeh, "Numerical study of a hemi-spherical cup in steady, unsteady, laminar and turbulent flow conditions with a vent of air at the top," Fluids Annual Review of Fluid Mechanics, vol. 28, pp. 361-387, 2008.

[11] M. J. Izadi and M. Dawoodian, "CFD analysis of drag coefficient of a parachute in steady and turbulent conditions in various Reynolds numbers," in Proceedings of the Fluids Engineering Division Summer Conference (FEDSM '09), pp. 2285-2293, ASME, Colorado, Colo, USA, August 2009.
[12] K. Takizawa, S. Wright, C. Moorman, and T. E. Tezduyar, "Fluid-structure interaction modeling of parachute clusters," International Journal for Numerical Methods in Fluids, vol. 65, no. 1-3, pp. 286-307, 2011.

[13] Y. Cao and C. Jiang, "Numerical simulation of the flow field around parachute during terminal descent," Aircraft Engineering and Aerospace Technology, vol. 79, no. 3, pp. 268-272, 2007.

[14] Y. Cao and H. Xu, "Parachute flying physical model and inflation simulation analysis," Aircraft Engineering and Aerospace Technology, vol. 76, no. 2, pp. 215-220, 2004.

[15] D. Ormières and M. Provansal, "Transition to turbulence in the wake of a sphere," Physical Review Letters, vol. 83, no. 1, pp. 8083, 1999. 

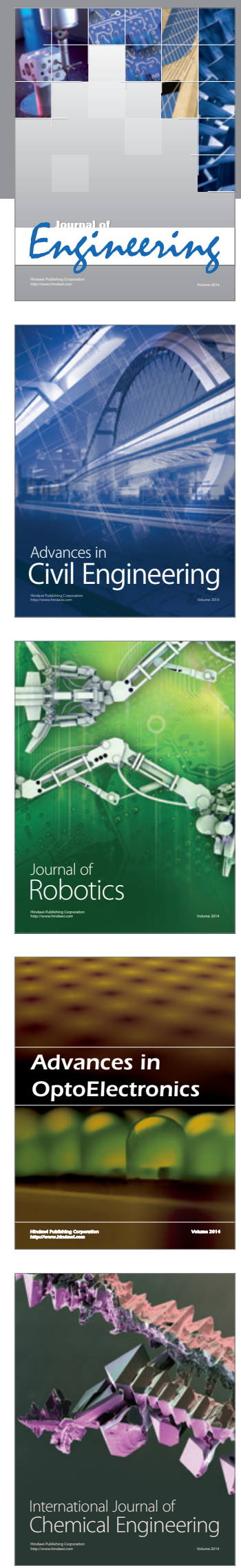

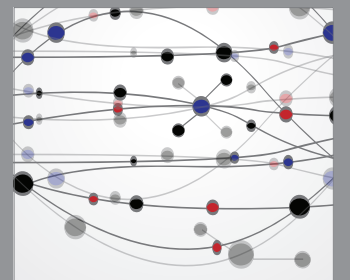

The Scientific World Journal
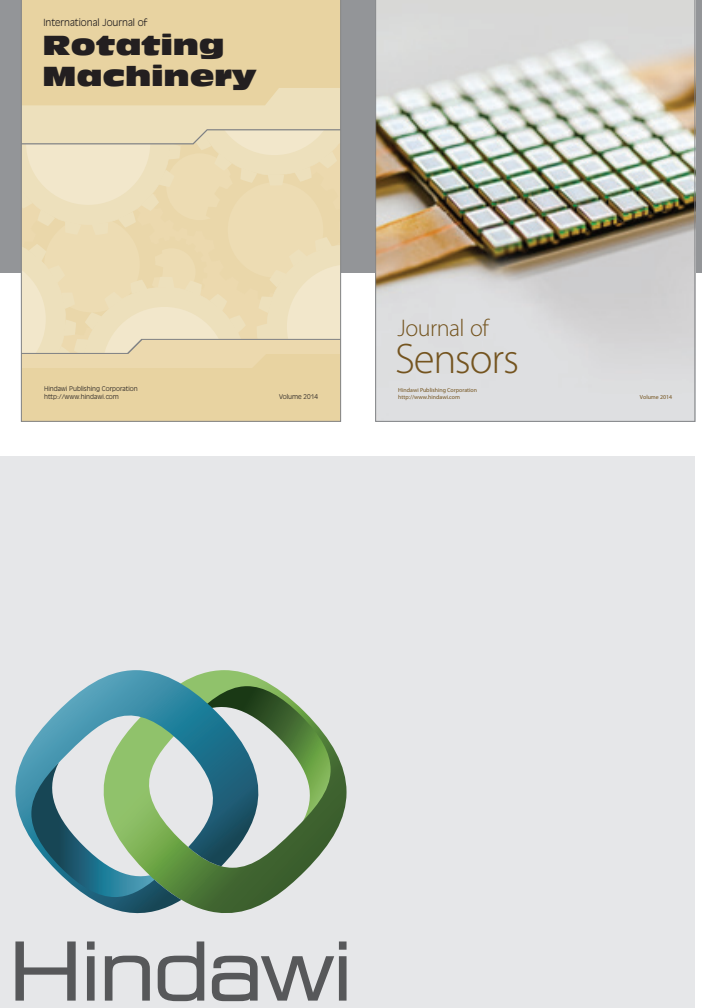

Submit your manuscripts at http://www.hindawi.com
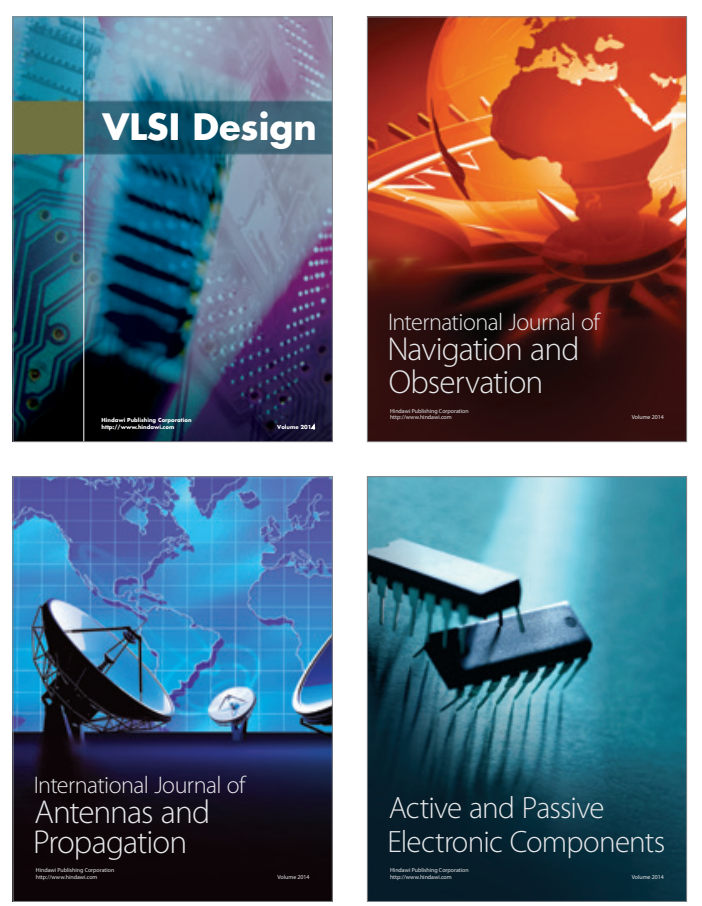
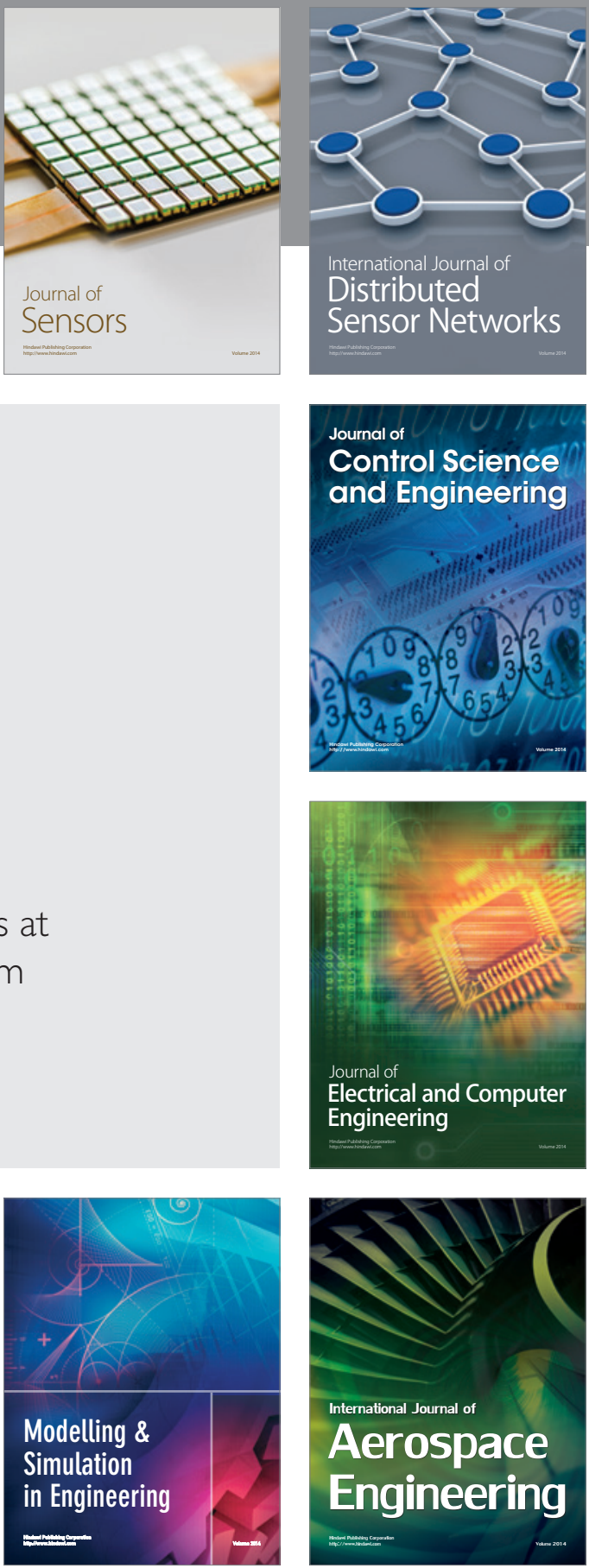

Journal of

Control Science

and Engineering
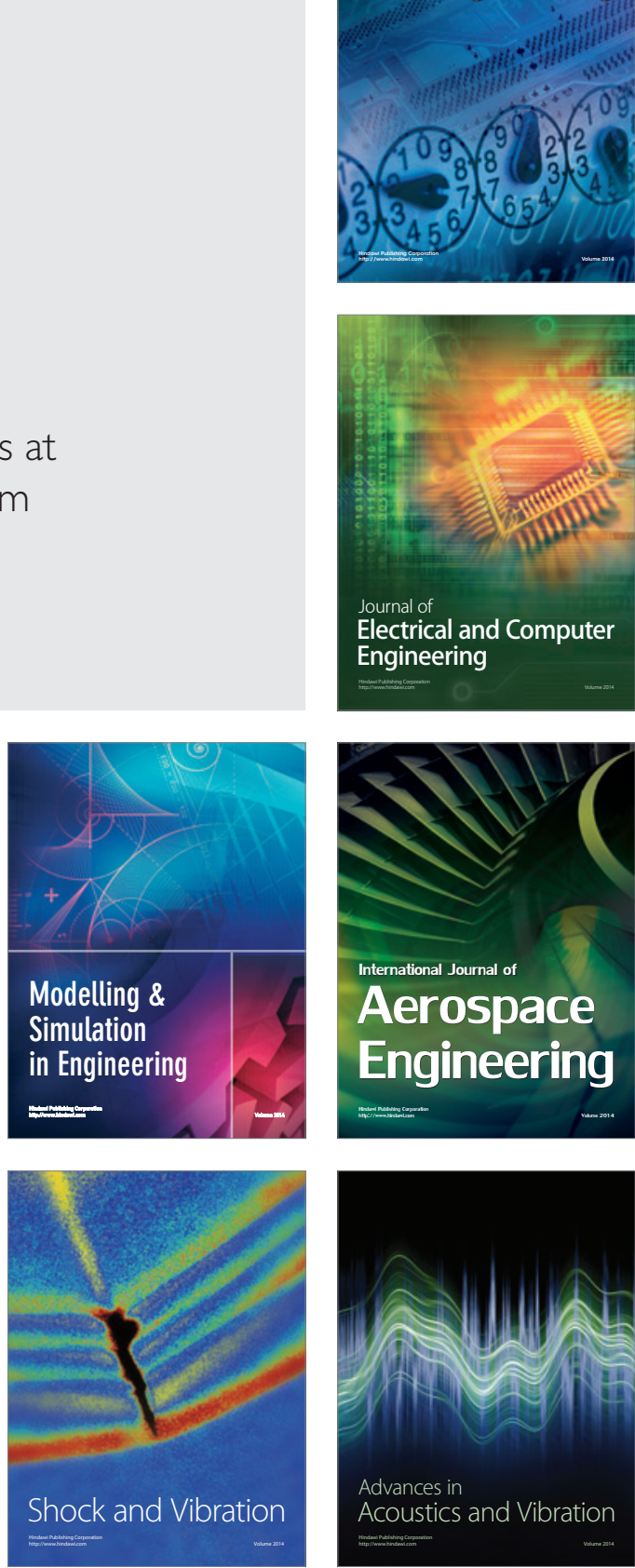